\title{
XCSF for Automatic Test Case Prioritization
}

\author{
Lukas Rosenbauer $^{1}$, Anthony Stein ${ }^{2}$, David Pätzel ${ }^{3}$ and Jörg Hähner ${ }^{3}$ \\ ${ }^{1}$ BSH Home Appliances, Im Gewerbepark B35, Regensburg, Germany \\ ${ }^{2}$ Artificial Intelligence in Agricultural Engineering, University of Hohenheim, Garbenstr. 9, Stuttgart, Germany \\ ${ }^{3}$ Organic Computing Group, University of Augsburg, Eichleitnerstr. 30, Augsburg, Germany
}

Keywords: Testing, Learning Classifier Systems, Evolutionary Machine Learning, Reinforcement Learning.

Abstract: Testing is a crucial part in the development of a new product. Due to the change from manual testing to automated testing, companies can rely on a higher number of tests. There are certain cases such as smoke tests where the execution of all tests is not feasible and a smaller test suite of critical test cases is necessary. This prioritization problem has just gotten into the focus of reinforcement learning. A neural network and an $\mathrm{XCS}$ classifier system have been applied to this task. Another evolutionary machine learning approach is the $\mathrm{XCSF}$ which produces, unlike XCS, continuous outputs. In this work we show that XCSF is superior to both the neural network and XCS for this problem.

\section{INTRODUCTION}

Evolutionary computation has lead to several advances in testing. Rodrigues et al. (2018) examined how a genetic algorithm (GA) can be used to generate test data. Nature inspired techniques have been exploited by Haga and Suehiro (2012) to automatically generate test cases. Another application is to mutate certain parts of the software to be tested in order to detect how effective tests are (Jia and Harman, 2008). The former has become its own field called mutation testing. Further GAs have been used to decide how resources should be allocated in order to ensure how reliability can be guaranteed (Dai et al., 2003).

Our use case is located in continuous integration (CI) that is a practice in software development to frequently integrate the work of each engineer. Thus big forks that are hard to merge can be avoided and software quality can be ensured. CI is made possible by using an automation tool such as Jenkins (Smart, 2011). Jenkins can be used to checkout source code, build it, test it, and deploy it. These steps are summarized as pipelines (see Figure 1). One execution of such a pipeline is called $C I$ cycle.

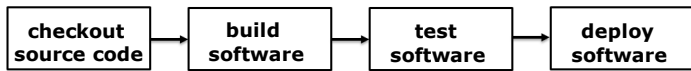

Figure 1: Example of a CI pipeline.
In this paper we concentrate solely on the testing stage during which tests from any testing level may run such as unit tests or even system tests. These can vary in terms of their duration and ability to detect failures. In some situations such as smoke testing it is not feasible to run all tests as there is not enough time (Dustin et al., 1999). Thus crucial tests must be selected to form a test suite whose execution time does not exceed a given time budget. The test suite should be adapted from cycle to cycle, reacting to code changes that influence the tests' outcomes. The desired test suite should be optimal in terms of its ability to find errors: Tests that can be expected to pass should be skipped whereas tests that would detect errors should be executed. Thus the tests are to be ranked according to their ability to find errors. The prioritization and time budget induce the test suite. The task of selecting such a prioritization and test suite optimally is referred to as adaptive test case selection problem (ATCS) (Spieker et al., 2017).

Spieker et al. (2017) and Rosenbauer et al. (2020) exploit the fact that the CI infrastructure saves the testing history (duration of tests, executions, test results). This information is used to construct a prioritization of the test cases. During each CI cycle the available test cases are parsed and ranked by reinforcement learning (RL) agents. An agent either gets a penalty or a reward, based on the outcome of the execution and the determined prioritization. 
Spieker et al. (2017) employ a neural network-based agent while Rosenbauer et al. (2020) utilize one based on the XCS classifier system (XCS) (Wilson, 1995).

$\mathrm{XCS}$ belongs to the family of learning classifier systems (LCS). LCSs are a framework of evolutionary rule-based machine learning methods (Urbanowicz and Browne, 2017). Research for LCS goes in multiple directions: mathematical examination as documented by Pätzel et al. (2019), reduction of runtime (Lanzi and Loiacono, 2010) or how the structure of LCSs can be adapted to improve learning performance (Stein et al., 2020). One such adaptation of $\mathrm{XCS}$ is called XCSF. It differs from XCS as it has a continuous output instead of a discrete one (Wilson, 2002). This is achieved by introducing polynomial models as predictors to the classifiers of XCSF.

During this work we apply XCSF to the ATCS problem. In order to do so, we examine three different data sets. This has lead to the following contributions:

- We show that the piece-wise function approximation principle of XCSF is an advantage over XCS. In all our experiments XCSF has an equal or better performance.

- Rosenbauer et al. (2020) conducted a preliminary study about the suitability of LCSs for ATCS. For this they considered the three data sets and reward functions that were used to benchmark the neural network of Spieker et al. (2017). The network was in some cases superior to XCS. Our XCSF approach is superior to the network in all but one cases. If another reward function is used, then $\mathrm{XCSF}$ is equal in terms of performance on this data set.

We continue this paper with a brief discussion of related work in Section 2. Afterwards we describe ATCS in general and its interpretation as a RL problem. Further we describe the policy that we want to use that is based on the approximation of a state-value function (Section 3). In Section 4 we describe XCS since XCSF is a mere extension of it. This is followed by a description of how XCSF evolved from XCS and how we adapted it for ATCS (Section 5). We benchmark our XCSF-based agent on three industrial data sets against the neural network of Spieker et al. (2017) and the XCS of Rosenbauer et al. (2020) in Section 6. We discuss possible future work in Section 7. We close this work with a conclusion (Section 8).

\section{RELATED WORK}

Several approaches outside RL already exist to prioritize test cases. Di Nardo et al. (2015); Mirarab et al.
(2012) prioritize based on the coverage of the test cases that requires a more detailed knowledge about the underlying software. Gligoric et al. (2015) analyse code dependencies to form a regression test suite. Kwon et al. (2014) apply information retrieval techniques to the source code and unit tests in order to form a test suite. An entire survey about test case prioritization was conducted by Marijan et al. (2013).

There are already several pure history-based approaches to form a prioritization (Park et al., 2008; Jung-Min Kim and Porter, 2002; Noguchi et al., 2015). The first RL-based approach that solely relies on historical data has been developed by Spieker et al. (2017). Spieker et al. (2017) performed a competitive study where they successfully compared their neural network with several non-RL methods. The RL approach ensures easy integration into existing development systems that enabled companies such as Netflix to use $\mathrm{it}^{1}$. Rosenbauer et al. (2020) conducted a preliminary study for the RL setting using XCS.

Epitropakis et al. (2015) developed a method to find a prioritization technique that intends to fulfill several criteria. There are also several approaches that are specialized for a specific form of testing such as automotive (Haghighatkhah, 2020), user interfaces (Nguyen et al., 2019) or production systems (Land et al., 2019).

From a machine learning point of view our work is deeply linked to LCS research. Applications range from traffic control (Tomforde et al., 2008), over distributed camera control (Stein et al., 2017) to manufacturing (Heider et al., 2020a). We are also not the first to apply XCSF to a real-world problem. It can be used to control robots (Stalph et al., 2009) or for image classification (Lee et al., 2012). It is also worth mentioning that XCSF is not the only LCS that provides a continuous output, for example SupRB's action space is also continuous (Heider et al., 2020b). However, we are first to our knowledge to apply $\mathrm{XCSF}$ to a testing use case.

\section{PROBLEM DESCRIPTION}

In order to be consistent with the previous literature, we follow the notation of Rosenbauer et al. (2020).

Let $T$ be a test case. It has an estimated duration of $d(T)$ and during CI cycle $i$ there is a total execution time of $C$ available. During each CI cycle, each test gets assigned a $\operatorname{rank}_{i}(T)$ which serves as a priority. Ranks are not unique (it is possible for two tests to get

\footnotetext{
${ }^{1} \mathrm{~A}$ corresponding article can be found here: Netflix techblog
} 
assigned the same rank). After this prioritization step, a schedule is created that takes into account the time budget available: The available tests are sorted descendingly by their ranks, then, tests are taken repeatedly from the start of the resulting list until the sum of the already selected tests' estimated durations just not yet exceeds the time budget. If there is not enough time to schedule all tests of one rank, then tests of this rank are selected uniformly at random until the termination criterion is met. Let $l_{i}(T)$ be the index of a test to bes executed $T$ in the schedule. The selected tests make up the test suite $\mathcal{T} S_{i}$ which is executed as part of CI cycle $i$. Let $\mathcal{T} \mathcal{S}_{i}^{\mathrm{f}}$ be the test cases in $\mathcal{T} \mathcal{S}_{i}$ that failed and $\mathcal{T S}_{i}^{\mathrm{t}, \mathrm{f}}$ be the set of failed tests if all available tests had been executed. The number of errors the test suite found relative to $\mathcal{T S} \mathcal{S}_{i}^{\mathrm{t}, \mathrm{f}}$ is then:

$$
p_{i}=\frac{\mathcal{T S}_{i}^{\mathrm{f}}}{\mathcal{T S}_{i}^{\mathrm{t}, \mathrm{f}}}
$$

A widespread metric for evaluating the quality of a test case prioritization is the normalized average percentage of faults detected (NAPFD) (Qu et al., 2007):

$$
\operatorname{NAPFD}\left(\mathcal{T S}_{i}\right)=p_{i}-\frac{\sum_{T \in \mathcal{T} \mathcal{S}_{i}^{\mathrm{f}}} l_{i}(T)}{\left|\mathcal{T} \mathcal{S}_{i}^{\mathrm{f}}\right| \cdot\left|\mathcal{T} \mathcal{S}_{i}\right|}+\frac{p_{i}}{2 \cdot\left|\mathcal{T} \mathcal{S}_{i}\right|}
$$

For NAPFD, high values are desired as small test suites that detect many failures result in such high values-especially if the failed tests were ranked high. On the other hand, either failed tests with a low rank or an increased number of high priority tests which passed without error decrease the NAPFD value. Thus, NAPFD measures the quality of both the prioritization as well as the test suite.

Using the definition of NAPFD we are now able to define the adaptive test case selection problem (ATCS):

$$
\begin{array}{ll}
\max & \operatorname{NAPFD}\left(\mathcal{T S} \mathcal{S}_{i}\right) \\
\text { subject to } & \sum_{T \in \mathcal{T S}_{i}} d(T) \leq C \\
& \mathcal{T} \mathcal{S}_{i} \subseteq \mathcal{T}_{i}
\end{array}
$$

where $\mathcal{T}_{i}$ denotes the set of available test during cycle $i$. Thus the goal is the choice of $\mathcal{T S}_{i}$ based on $\mathcal{T}_{i}$.

Similar to Spieker et al. (2017) and Rosenbauer et al. (2020), we intend to solve ATCS using RL which leads to the workflow described in Figure 2. The following paragraphs describe several possible reward functions as well as the state and action spaces.

A reasonable thought is to try using the NAPFD metric as a reward function for an agent. However, in practice this would force us to always execute all tests ( $p_{i}$ is needed for its computation) which we explicitly want to avoid. Thus Spieker et al. (2017) came up with three alternative reward functions. The first is the failure count reward

$$
r_{i}^{\mathrm{fc}}(T)=\left|\mathcal{T S}{ }_{i}^{\mathrm{f}}\right|
$$

which does not distinguish between individual tests as all receive the same reward. A more fine-grained approach is the test failure reward,

$$
r_{i}^{\mathrm{tcf}}(T)= \begin{cases}1-v_{i}(T) & T \in \mathcal{T} \mathcal{S}_{i} \\ 0 & \text { otherwise }\end{cases}
$$

where $v_{i}(T)$ is the binary verdict of $T$ during cycle $i$ with 0 indicating 'test failed' and 1 indicating either of 'test passed' or 'test not executed due to the time restriction'. The advantage of $r_{i}^{\mathrm{tcf}}$ over $r_{i}^{\mathrm{fc}}$ is that it rewards tests individually based on their outcome. However, it does not take the test's rank into account which has an impact on NAPFD. Thus Spieker et al. (2017) came up with the time ranked reward

$$
r_{i}^{\mathrm{trk}}(T)=\left|\mathcal{T S}_{i}^{\mathrm{f}}\right|-v_{i}(T) \cdot \sum_{\substack{t_{k} \in \mathcal{T} \mathcal{S}_{i}^{\mathrm{f}} \\ \operatorname{rk}(T)<\operatorname{rk}\left(t_{k}\right)}}
$$

which still gives all failed test cases the same reward but does distinguish the non-failed tests. These are punished by the number of failed tests with a lower rank. For example, a passed test that was correctly ranked with a low priority will get a high reward and a passed test with a high rank leads to a penalty. In general, we denote the reward received at time $t$ as $r(t)$.

The problem's state space is defined as follows:

$$
S=[0, C] \times\{0,1\}^{k} \times[0,1]
$$

The state (a test case) contains the approximated $d u$ ration (a real number between 0 and $C$ ), the testing history (a binary vector of length $k$ ), and the time of the last execution relative to the entire testing history (a real number between 0 and 1). The hyperparameter $k$ indicates how many previous outcomes of the test case are given to the agent. If there are not yet $k$ test results available, the missing entries are filled with zeroes. States are vectors of dimension $k+2$; we denote the state at time $t$ by $s(t)$.

The action space for both our XCSF-based solution as well as the network of Spieker et al. (2017) is $\mathbb{R}$ whereas the XCS-based agent of Rosenbauer et al. (2020) used an action space of $\{0,1, \ldots, 45\}$. We write the action performed at time $t$ as $a(t)$.

It is worth mentioning that the RL interpretation of ATCS slightly differs from the most common temporal difference learning (TD) setting as, here, first all tests are ranked and only after the execution of the tests of $\mathcal{T}_{i}$ are rewards distributed. In TD, sequences 


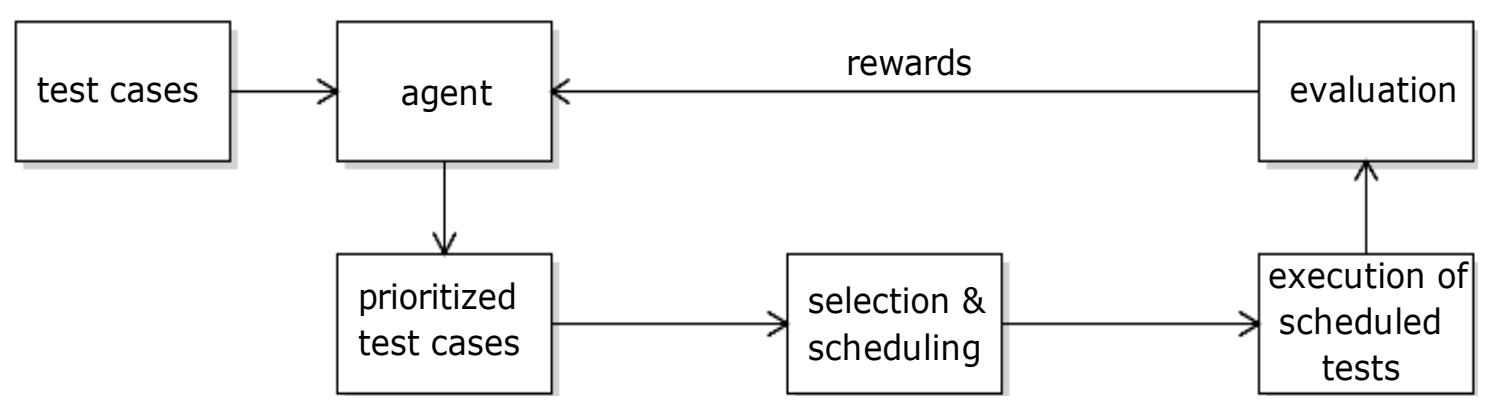

Figure 2: Workflow for solving ATCS using RL.

of states, actions and rewards are usually observed until a terminal state is observed:

$$
s(1), a(1), r(1), s(2), a(2), r(2), \ldots
$$

For ATCS, the sequence instead has the form

$$
s(1), a(1), s(2), a(2) \ldots, s(n), a(n), r(1), r(2) \ldots r(n)
$$

with episodes corresponding to CI cycles (the terminal state being the end of the cycle) which means that the agent's actions do not determine the length of episodes. Also, the selection of states encountered during an episode is fixed as well as the test cases to be ranked are known. However, it is worth mentioning that the environment may change from cycle to cycle as new bugs may be introduced to the software or existing ones may be fixed (leading to additional failed or succeeding tests, respectively).

A method often used for solving RL problems is to approximate the state-action-value function (often called Q-function) which assigns a measure of value to each state-action pair $(s, a)$ (e.g. the expected return when action $a$ is performed in $s$ and a certain policy followed thereafter). Instead, our system approximates a state-value function $V(\cdot)$ for the following policy $\pi$ :

$$
\pi(s)=\hat{\mathrm{V}}(s)
$$

where $\hat{\mathrm{V}}(\cdot)$ is the approximation of $V(\cdot)$. This policy follows a simple heuristic: A test case's priority should correspond to the value that can be achieved or in other words if a test case (i. e. a state) has a high value then it should have a high priority. $\hat{\mathrm{V}}(\cdot)$ estimates the reward that will be received if that policy is applied. Thus our RL approach can also be seen as a form of regression.

\section{XCS CLASSIFIER SYSTEM}

A learning classifier system (LCS) roughly consists of a population of rules, a learning mechanism for them and an evolutionary heuristic to optimize their localization in input space (usually a GA). The rules are called classifiers and we denote one such rule by cl. Each classifier proposes an action cl. $a$ to be executed if the conditions it specifies are met by the state $s(t)$. Conditions can vary in their form depending on the problem. For example, a condition could be a Boolean predicate that a value is in an interval. Further, each classifier tracks certain quality measures that can be used to decide which rules of the population should be applied.

A widespread LCS is the XCS classifier system (XCS) which was introduced by Wilson (1995). A classifier in XCS tracks how often it has been applied. This is coined the classifier's experience. Further parameters include the classifier's predicted payoff $c l . p$ which estimates the return to be expected if the rule is applied, an error estimate for that prediction cl.e and a niche-relative payoff prediction accuracy called fitness cl. $f$.

Whenever XCS classifier system encounters a new state $s(t)$, it searches its population for classifiers whose conditions are met (this is called matching). If not enough classifiers are found then new ones are created probabilistically whose conditions match $s(t)$. This process is called covering. The found and newly created classifiers make up the match set $M_{t}$. Based on the match set XCS computes a quality measure called system prediction, the fitness-weighted sum of predicted payoffs, for each action in available in the match set:

$$
\frac{\sum_{\mathrm{cl} \in M_{t}, \mathrm{cl} \cdot a=a} \mathrm{cl} \cdot p \cdot \mathrm{cl} \cdot f}{\sum_{\mathrm{cl} \in M_{t}, \mathrm{cl} \cdot a=a} \mathrm{cl} \cdot f}
$$

These values make up the prediction array $\mathrm{PA}_{t}$ which is used as a base for decision-making. XCS usually either chooses the action at random to explore the environment or according to the highest system prediction value. After it has decided for an action it selects all classifiers of $M_{t}$ that propose the chosen action and summarizes them in the action set $A_{t}$. After the execution of the chosen action, XCS receives a reward which is used to calculate the update target $P$ :

$$
P=r(t)+\gamma \cdot \max \left(\mathrm{PA}_{t+1}\right)
$$


where $\gamma$ is the usual reinforcement learning discounting factor. It is used to update the classifiers of the previous action set $A_{t-1}$ such that each classifier learns to model the expected return received when its action is executed for states that match its conditions.

XCS additionally employs a genetic algorithm to optimize the conditions of the classifiers available. Its task is to find an optimal partition of the classifiers for the problem surface by adapting their localization. The heuristic creates new classifiers based on existing ones of the population by applying crossover and mutation operators on their conditions. The GA is used periodically (e. g. after every tenth state encountered).

The population of XCS has a fixed capacity. After each execution of the GA and the matching process, its size is checked. If the population has too many elements then XCS deletes classifiers at random but proportional to their quality. We call this deletion mechanism pruning.

We describe the basic workflow of XCS in Algorithm 1. The function choose_action employs a $\varepsilon$ greedy policy to select the next action (Butz and Wilson, 2001). We keep the description of XCS at this abstract level but recommend to read Urbanowicz and Browne (2017) where LCSs are described in detail.

Algorithm 1: XCS workflow based on Butz and Wilson (2001).

input : $s(t), r(t-1)$

$1 M_{t}=$ matching(population, $s(t)$ )

$2 \mathrm{PA}_{t}=$ prediction_array $\left(M_{t}\right)$

$3 a=$ choose_action $\left(\mathrm{PA}_{t}\right)$

$4 A_{t}=$ get_action_set $\left(M_{t}, a\right)$

5 execute $a$, observe $r(t)$

$6 \mathrm{P}=r(t-1)+\gamma \max \left(\mathrm{PA}_{t}\right)$

7 // if non-empty

8 update $A_{t-1}$ by $\mathrm{P}$

$9 A_{t-1}=A_{t}$

10 if $G A$ should run then

11 run GA on $A_{t}$

12 prune population

Rosenbauer et al. (2020) adapted XCS to the problem as it originally follows the usual TD learning scheme based on the sequence of states, actions and rewards given in Equation 8 and not the sequence given in Equation 9. In order to adapt XCS to the problem they introduced a batch update rule. Instead of using the maximum system prediction of the succeeding state, they use the average maximum system prediction of the states of the succeeding CI cycle to compute $P$ :

$$
P=r(t)+\gamma \cdot \frac{\sum_{j \in e_{i+1}} \max \left(\mathrm{PA}_{j}\right)}{\left|\mathcal{T}_{i+1}\right|}
$$

where $e_{i+1}$ denotes the time steps of the cycle $i+1$. Further, the classifiers are always updated at the end of a cycle and not between two states. In our later experiments we simply call this variant XCS.

Additionally, Rosenbauer et al. (2020) examined whether a experience replay version of XCS can improve results for ATCS. However, this proved to be detrimental in most cases which is why we do not include that version in our later experiments.

\section{FROM XCS TO XCSF}

XCS as proposed by Wilson (1995) produces a discrete output (the actions). Some problems, however, have a more continuous nature. Thus Wilson (2002) adapted XCS to also provide a real-valued output which resulted in the XCSF classifier system, a general function approximator. In our use case we use XCSF to approximate $V(\cdot)$ as already mentioned in Section 3 which enables us to use continuous actions.

In contrast to standard XCS, a classifier in XCSF does not have a scalar prediction. Instead its prediction is modelled as a function that approximates the expected payoff. Here we model the classifier's prediction function as follows:

$$
c l . p(s)=w_{0}+\sum_{i=1}^{k+2} w_{i} \cdot s_{i}
$$

where $s$ is an element of the state space and $w_{i}$ represent real-valued weights. Initially the weights are set randomly. The weights are updated using a gradientdescent-based method called modified delta rule (Wilson, 2002):

$$
\Delta w_{i}=\frac{\eta}{\|\tilde{s}\|^{2}}(r-c l \cdot p(s)) \tilde{s}_{i}
$$

The old estimation of $w_{i}$ is simply updated by adding $\Delta w_{i}$. Here $\eta$ denotes the learning rate which controls how strongly a new experience can influence the current estimator. The vector $\tilde{s}$ is the same as the state vector $s$ but with an additional of 1 for fitting the intercept:

$$
\tilde{s}=\left(1, s_{1}, s_{2}, \ldots, s_{k+2}\right)^{T}
$$

Further, $\|\tilde{s}\|$ is the euclidean norm of $\tilde{s}$ and $r$ is the reward that corresponds to the state $s$. Thus we do not use $P$ of Equation 12 as an update target, but the actual reward.

XCSF classifiers only maintain a dummy action (in order to stick to the original workflow of XCS). Hence XCSF computes only one system prediction as follows:

$$
\frac{\sum_{\mathrm{cl} \in M_{t}} \mathrm{cl} \cdot p(s(t)) \cdot \mathrm{cl} \cdot f}{\sum_{\mathrm{cl} \in M_{t}} \mathrm{cl} \cdot f}
$$


which is used as estimation for the function to be approximated (in our case $V(s(t))$ ). We directly use the estimation as action as we employ the policy of Equation 10.

For XCSF, the match set is equal to the action set. Hence all matching classifiers are updated whenever a reward is received. Further $M_{t}$ is updated using $r(t)$.

In our experiments we employ a batch update mode as we only receive rewards at the end of a CI cycle. Thus during the prioritization of tests we solely choose ranks and save the action sets that will be updated as described in Algorithm 2. At the end of the cycle we update the collected action sets, run the GA and prune the population (see Algorithm 3).

It is worth mentioning that we further differ from Wilson (2002) as the states $s$ also contain binary variables instead of exclusively real numbers. We can still fit our linear models cl.p(.) since gradient-descent based methods such as the modified delta rule only require continuous weights and not continuous states (as the weights are optimized) (Aggarwal, 2020).

\begin{tabular}{l}
\hline Algorithm 2: Action selection of XCSF. \\
\hline input : state $s$ \\
output: action $a$ \\
$1 M_{t}=$ matching(population, $\left.s\right)$ \\
$2 a=\frac{\sum_{\mathrm{cl} \in M_{t}} \mathrm{cl} \cdot p(s) \cdot \mathrm{cl} . f}{\sum_{\mathrm{cl} \in M_{t}} \mathrm{cl} \cdot f}$ \\
$3 A_{t}=M_{t}$ \\
4 cycle_action_sets.append $\left(A_{t}\right)$ \\
5 return $a$ \\
\hline
\end{tabular}

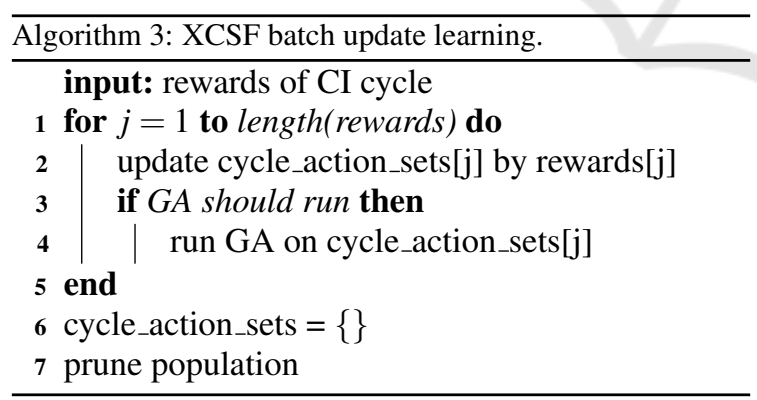

\section{EVALUATION}

In our experiments we compare our batch update XCSF with the XCS variant of Rosenbauer et al. (2020) and the artificial neural network approach of Spieker et al. (2017). We reuse the three data sets of Spieker et al. (2017). The first two are from a Scandinavian robot company and called $A B B$ paint control and $A B B I O F / R O L$. Additionally the Google shared data set of test suite results (GSDTSR) is considered.

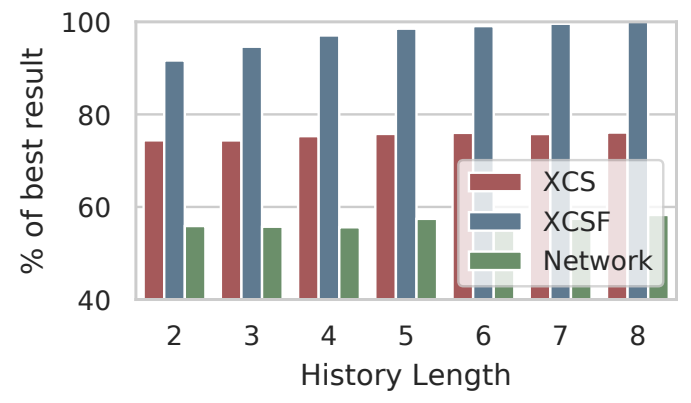

Figure 3: Evaluation of the history length's influence on performance. The y-axis displays the average NAPFD values of the methods in relation to the best average NAPFD achieved.

Table 1: Examined data sets.

\begin{tabular}{cccc}
\hline & $\begin{array}{c}\text { paint } \\
\text { control }\end{array}$ & IOF/ROL & GSDTSR \\
\hline CI cycles & 312 & 320 & 336 \\
test cases & 114 & 2,086 & 5,555 \\
verdicts & 25,594 & 30,319 & $1,260,617$ \\
failed & $19.36 \%$ & $28.43 \%$ & $0.25 \%$ \\
\hline
\end{tabular}

Table 1 gives a brief overview about the structure of these data sets. All three data sets vary in terms of their test cases, verdicts, and amount of failed tests. Thus we can examine the three methods on a broader scope.

Table 2: P-Values for paired Student t-tests (rounded to the fifth decimal). Values below 0.05 are marked bold. The null hypothesis is that XCS is superior to XCSF.

\begin{tabular}{cccc}
\hline & Paint Control & IOF/ROL & GSDTSR \\
\hline$r^{\text {fc }}$ & $\mathbf{0 . 0 0 5 2 3}$ & 0.25042 & $\mathbf{0 . 0 0 0 2}$ \\
$r^{\text {tcf }}$ & 0.30446 & 0.32404 & $\mathbf{0 . 0 0 0 9 1}$ \\
$r^{\text {trk }}$ & $\mathbf{0 . 0 0 1 9 2}$ & 0.14235 & $\mathbf{0 . 0 0 0 0 1}$ \\
\hline
\end{tabular}

Table 3: P-Values for paired Student t-tests (rounded to the fifth decimal). Values below 0.05 are marked bold. The null hypothesis is that the neural network is superior to XCSF.

\begin{tabular}{cccc}
\hline & Paint Control & IOF/ROL & GSDTSR \\
\hline$r^{\mathrm{fc}}$ & $\mathbf{0 . 0}$ & $\mathbf{0 . 0 1 4 7 3}$ & $\mathbf{0 . 0}$ \\
$r^{\mathrm{tcf}}$ & 0.99999 & $\mathbf{0 . 0 0 0 1 5}$ & $\mathbf{0 . 0}$ \\
$r^{\mathrm{trk}}$ & $\mathbf{0 . 0}$ & $\mathbf{0 . 0 0 0 7 8}$ & $\mathbf{0 . 0}$ \\
\hline
\end{tabular}

For the XCS-based agent and the neural network we can rely on the original implementation. Both were also benchmarked on the same data sets that we consider. We adopted the original hyperparameters proposed by Spieker et al. (2017) and Rosenbauer et al. (2020) for each respective agent, respectively. This way, we ensure a fair comparison with our approach. 


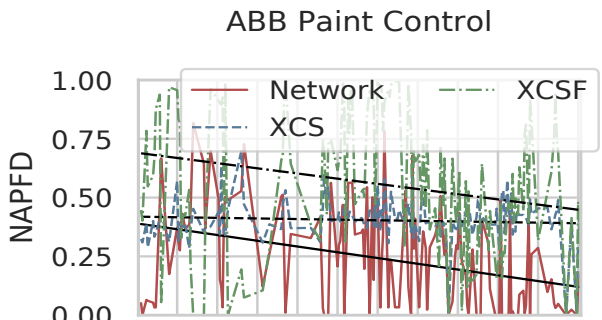

ABB IOF/ROL

(a) Failure Count Reward
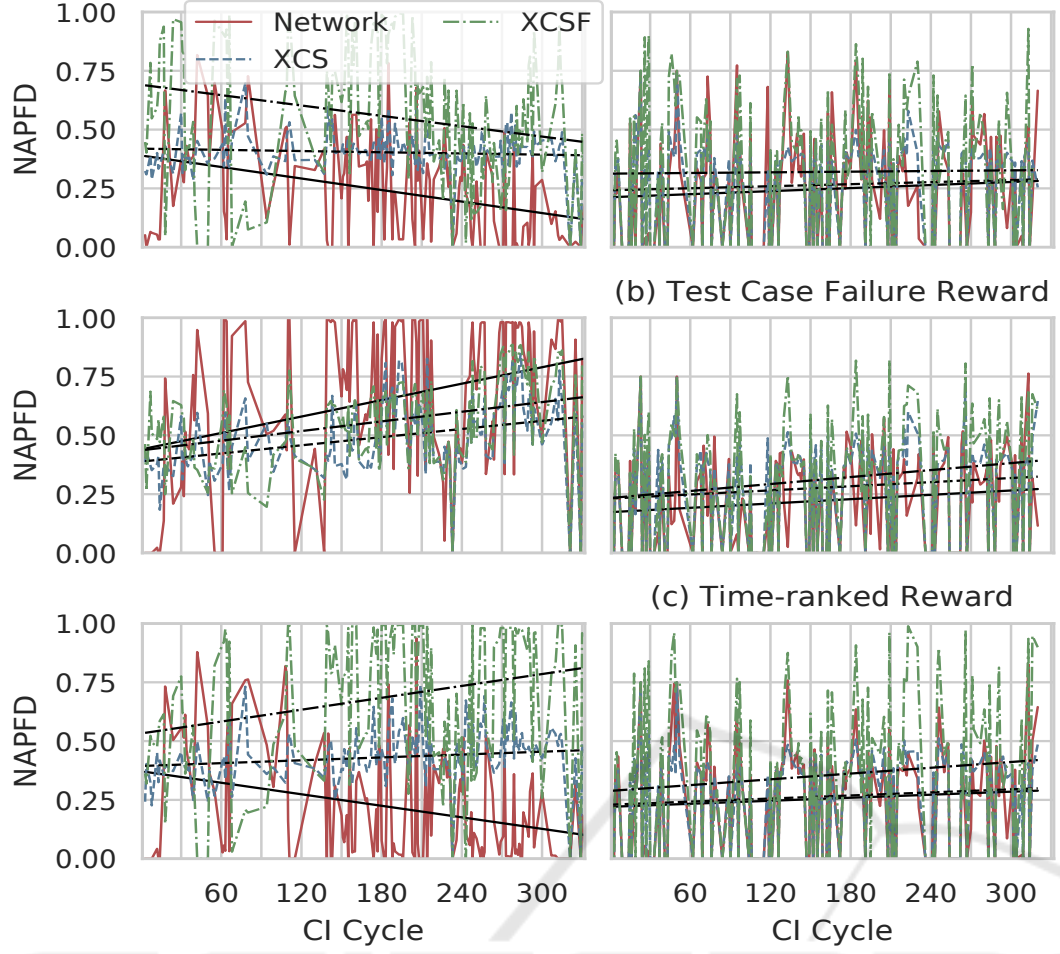

(b) Test Case Failure Reward

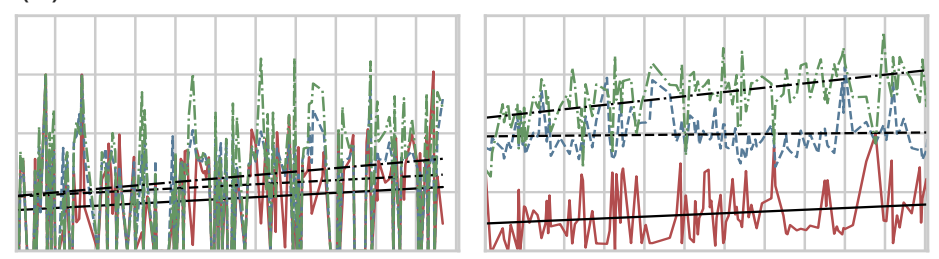

(c) Time-ranked Reward

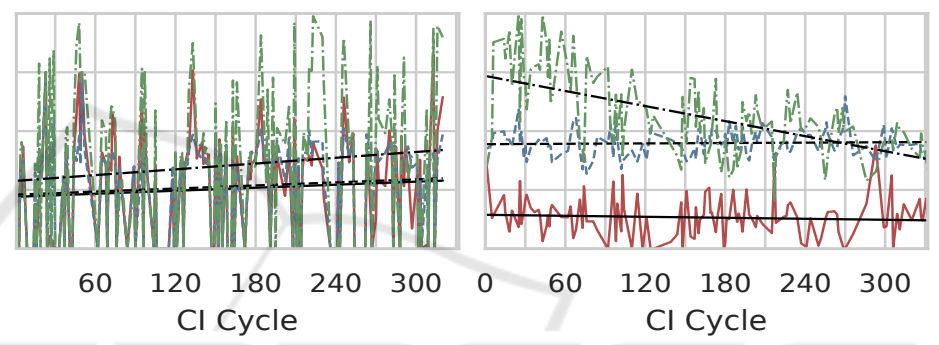

Figure 4: Comparison of XCSF with XCS and the neural network.

To our knowledge there is no XCSF library for states that both have binary and real valued components. Hence we had to implement our own XCSF which we did in Python $3^{2}$. We modelled the conditions for the real values as intervals and for the binary parts of the states we follow the original XCS and use ternary conditions (Wilson, 1995). For the GA we use a roulette wheel selection-based on the classifier's fitness. For the crossover of the ternary conditions we apply a one-point crossover and for the intervals and weights of $c l . p(\cdot)$ an arithmetic one. For the mutation of the ternary conditions we follow Butz and Wilson (2001) and for the interval-based conditions and weights of $c l . p(\cdot)$ we apply a random mutation (i.e. choosing an entirely random interval). We don't perform any form of subsumption.

We set the learning rate $\eta$ to 0.1 . We draw the initial weights of $c l . p(\cdot)$ during covering and mutation uniform at random from $[-10,10]$. For the remaining hyperparameters we follow the notation of Butz and Wilson (2001). The population capacity $N$ is 2000. $\alpha$ and $\beta$ are set to $0.15, v$ is $5, \theta_{G A}$ is $25, \mu$ is 0.025 . The initial error $\varepsilon_{I}$ and initial fitness $f_{I}$ of the classifiers is set to $0 . \theta_{\text {del }}$ is $20, \theta_{\text {sub }}$ is $20, \chi$ is $0.75, p_{\text {exp }}$ is 0.2 and

\footnotetext{
${ }^{2}$ source code: https://github.com/LagLukas/xcsf_atcs
}

$\varepsilon_{0}$ is 0.01 . During covering we set a binary condition to \# with a probability of 0.33 .

We perform thirty i.i.d. runs for every experiment and display the averaged results. We can simulate the real use case as we have the results of all test cases for every CI cycle. Thus we can use the reward functions of Section 3 whilst being able to evaluate the performance of the methods in terms of NAPFD.

In our first experiment we evaluate the influence of the history length $k$ on the performance. We examine the lengths $k=2,3, . ., 8$. For all methods we use the time ranked reward function. The results can be seen in Figure 3. The $\mathrm{x}$-axis shows the values for $k$ and the $y$-axis shows the average NAPFD values of the methods relative to the best. XCSF clearly exceeds the two RL agents for this combination of reward function and data set. We verified this visual observation with a series of one-sided paired Student$t$ tests which were significant. We further verified the necessary condition of normal distributed data with Shapiro-Wilk tests. For these tests we used a significance level of 0.05 .

Furthermore we could observe that a higher value of $k$ is beneficial for XCSF. Thus we will use a history length of 8 in the upcoming experiments. For the other agents the history length does not seem to affect 
their performance too much. However, this is only one out of nine possible combinations of reward function and data set. Hence we consider now all three data sets and reward functions in order to examine the performance of XCSF on a wider scope.

The results are displayed in Figure 4. Each column contains the results for one data set and each row represents the experimental outcome for one reward function. Each plot contains a trendline for every examined agent next to the averaged results.

We provide additional statistical tests in Table 2 and 3 as we deem a purely visual evaluation as insufficient. Especially as trendlines can easily be disturbed by statistical outliers. The tables contain p-values of paired Student-t tests where we marked p-values below 0.05 as significant. The necessary condition of normal distributed data was confirmed by ShapiroWilk tests (using a significance level of 0.05).

On the paint control data set (column 1) we can see that XCSF is superior to XCS (in terms of the trendline) for all reward functions but the performance, if the failure count reward is used, seems to decline over time. Statistical tests can confirm XCSF's superiority for the failure count reward and the time ranked reward. For the test case failure reward the p-value is not significant and thus we performed an additional t-test to test the nullhypothesis that XCSF is superior to XCS. The latter test was also not significant and thus we deem both systems equivalent for this combination of data set and reward function. However, we can observe the best performance for XCSF for the time ranked reward and there it clearly exceeds XCS regardless of the reward function in terms of NAPFD. XCSF is also able to outperform the neural network in two out of three cases. Visually we cannot determine if XCSF combined with the time ranked reward or the network combined with the test case failure reward is better suited for this data set or vice versa. Additional paired Student-t tests could also not reveal which approach is superior. Hence we deem both methods as equivalent for this problem.

For the IOF/ROL data (column 2) the trendlines indicate that XCSF is superior to XCS but statistical tests cannot confirm this observation (see Table 2). We additionally tested the nullhypothses that XCSF is superior to XCS and we could also not reject these. Hence we deem XCS and XCSF equivalent on this data set. On the other hand the statistical evaluation shows that XCSF is superior to the neural network. However, all three approaches have difficulties learning a good policy on this data set as the NAPFD results are generally worse than on the paint control data set.

Column 3 displays the results for the GSDTSR data set. For the failure count reward and the time ranked reward the performance of XCSF seems to decline over time and finally falls behind XCS. Due to the high NAPFD values for most CI cycles the statistical tests state that XCSF is better. However, visually the best approach for this data set is XCSF combined with the test case failure reward. This combination does not only outperform the XCS but also the neural network for all three reward functions (in terms of NAPFD). Furthermore it is also the only setting where we can observe a positive slope for the trendline. Spieker et al. (2017) and Rosenbauer et al. (2020) deemed this as rather unlikely to achieve as the data set contains very few failed tests.

We also observed that the structure of the data set should also be considered for the choice of the reward function for XCSF (Hamid and Braun, 2019). If the data set contains very few failed tests then the time ranked reward function proofs detrimental (see GSDTSR) but if it contains a certain amount of failures then it seems to be a good choice (see ABB data sets).

\section{FUTURE WORK}

We further want to boost the results of XCSF by using interpolation (Stein et al., 2018; Stein et al., 2016). The application of interpolation to certain parts of $\mathrm{XCSF}$ such as the GA can improve learning efficiency.

Another new direction in machine learning is to generate new experiences from previous ones (also by applying interpolation) and use them for training. von Pilchau et al. (2020) showed in a preliminary study that this proofs useful for artificial neural networks. The same could be the case for LCSs such as XCSF.

Further, we only applied linear functions for the classifiers. Lanzi et al. (2005) showed that polynomial functions of higher order such as cubic ones can also be beneficial for XCSF.

Furthermore we think that the performance of XCSF can be improved by using more information such as changelogs, git diffs or additional test metadata.

\section{CONCLUSION}

During this work we evaluated the test case prioritization problem coined adaptive test case selection problem (ATCS). Recently it has been interpreted as reinforcement learning problem and two agents are known in literature; an XCS-based one (Rosenbauer 
et al., 2020) and an approach using an artificial neural network-based (Spieker et al., 2017).

We used a XCSF-based agent and employed a simple heuristic: a test case of high value should have a high priority. Hence we used XCSF to approximate a state-value function $V(\cdot)$ and interpreted the approximated values as actions.

We benchmarked our agent on three different data sets using three reward functions. In our comparison, $\mathrm{XCSF}$ was in 8 out of 9 cases superior to the neural network. For a single combination of reward function and data set XCSF was inferior. However, if the best combinations of reward functions and agent are considered on the data set then both approaches are equal.

Our experiments showed that the continuous output leads to a performance boost compared to XCS as $\mathrm{XCSF}$ was in all nine cases considered either superior or had an equivalent performance. Thus we recommend to use XCSF for ATCS.
Haghighatkhah, A. (2020). Test case prioritization using build history and test distances: an approach for improving automotive regression testing in continuous integration environments.

Hamid, O. H. and Braun, J. (2019). Reinforcement Learning and Attractor Neural Network Models of Associative Learning, pages 327-349. Springer International Publishing, Cham.

Heider, M., Pätzel, D., and Hähner, J. (2020a). Towards a Pittsburgh-Style LCS for Learning Manufacturing Machinery Parametrizations. In Proceedings of the 2020 Genetic and Evolutionary Computation Conference Companion, GECCO '20, page 127-128, New York, NY, USA. Association for Computing Machinery.

Heider, M., Pätzel, D., and Hähner, J. (2020b). SupRB: A Supervised Rule-based Learning System for Continuous Problems.

Jia, Y. and Harman, M. (2008). Constructing Subtle Faults Using Higher Order Mutation Testing. In 2008 Eighth IEEE International Working Conference on Source Code Analysis and Manipulation, pages 249 - 258.

Jung-Min Kim and Porter, A. (2002). A history-based test prioritization technique for regression testing in resource constrained environments. In Proceedings of the 24th International Conference on Software Engineering. ICSE 2002, pages 119-129.

Kwon, J., Ko, I., Rothermel, G., and Staats, M. (2014). Test Case Prioritization Based on Information Retrieval Concepts. In 2014 21st Asia-Pacific Software Engineering Conference, volume 1, pages 19-26.

Land, K., Cha, S., and Vogel-Heuser, B. (2019). An Approach to Efficient Test Scheduling for Automated Production Systems. 2019 IEEE 17th International Conference on Industrial Informatics (INDIN), $1: 449-454$.

Lanzi, P. L. and Loiacono, D. (2010). "Speeding Up Matching in Learning Classifier Systems Using CUDA". In Bacardit, J., Browne, W., Drugowitsch, J., BernadóMansilla, E., and Butz, M. V., editors, Learning Classifier Systems, pages 1-20, Berlin, Heidelberg. Springer Berlin Heidelberg.

Lanzi, P. L., Loiacono, D., Wilson, S. W., and Goldberg, D. E. (2005). Extending xcsf beyond linear approximation. In Proceedings of the 7th Annual Conference on Genetic and Evolutionary Computation, GECCO '05, page 1827-1834, New York, NY, USA. Association for Computing Machinery.

Lee, P., Teng, Y., and Hsiao, T.-C. (2012). XCSF for Prediction on Emotion Induced by Image Based on Dimensional Theory of Emotion. In Proceedings of the 14th Annual Conference Companion on Genetic and Evolutionary Computation, GECCO '12, page 375-382, New York, NY, USA. Association for Computing Machinery.

Marijan, D., Gotlieb, A., and Sen, S. (2013). Test Case Prioritization for Continuous Regression Testing: An Industrial Case Study. In 2013 IEEE International Conference on Software Maintenance, pages 540-543.

Mirarab, S., Akhlaghi, S., and Tahvildari, L. (2012). SizeConstrained Regression Test Case Selection Using 
Multicriteria Optimization. IEEE Transactions on Software Engineering, 38(4):936-956.

Nguyen, A., Le, B., and Nguyen, V. (2019). Prioritizing Automated User Interface Tests Using Reinforcement Learning. In Proceedings of the Fifteenth International Conference on Predictive Models and Data Analytics in Software Engineering, PROMISE'19, page 56-65, New York, NY, USA. Association for Computing Machinery.

Noguchi, T., Washizaki, H., Fukazawa, Y., Sato, A., and Ota, K. (2015). History-Based Test Case Prioritization for Black Box Testing Using Ant Colony Optimization. In 2015 IEEE 8th International Conference on Software Testing, Verification and Validation (ICST), pages $1-2$.

Park, H., Ryu, H., and Baik, J. (2008). Historical ValueBased Approach for Cost-Cognizant Test Case Prioritization to Improve the Effectiveness of Regression Testing. In 2008 Second International Conference on Secure System Integration and Reliability Improvement, pages 39-46.

Pätzel, D., Stein, A., and Hähner, J. (2019). A Survey of Formal Theoretical Advances Regarding XCS. In Proceedings of the Genetic and Evolutionary Computation Conference Companion, GECCO '19, pages 1295-1302, New York, NY, USA. ACM.

Qu, X., Cohen, M. B., and Woolf, K. M. (2007). Combinatorial Interaction Regression Testing: A Study of Test Case Generation and Prioritization. In 2007 IEEE International Conference on Software Maintenance, pages $255-264$.

Rodrigues, D. S., Delamaro, M. E., Corrêa, C. G., and Nunes, F. L. S. (2018). Using Genetic Algorithms in Test Data Generation: A Critical Systematic Mapping. ACM Comput. Surv., 51(2).

Rosenbauer, L., Stein, A., Maier, R., Pätzel, D., and Hähner, J. (2020). XCS as a Reinforcement Learning Approach to Automatic Test Case Prioritization. In Proceedings of the Genetic and Evolutionary Computation Conference Companion, GECCO '20.

Smart, J. F. (2011). Jenkins: The Definitive Guide. O'Reilly, Beijing.

Spieker, H., Gotlieb, A., Marijan, D., and Mossige, M. (2017). Reinforcement Learning for Automatic Test Case Prioritization and Selection in Continuous Integration. In Proceedings of the 26th ACM SIGSOFT International Symposium on Software Testing and Analysis, ISSTA 2017, page 12-22, New York, NY, USA. Association for Computing Machinery.

Stalph, P. O., Butz, M. V., and Pedersen, G. K. M. (2009). Controlling a Four Degree of Freedom Arm in 3D Using the XCSF Learning Classifier System. In Mertsching, B., Hund, M., and Aziz, Z., editors, KI 2009: Advances in Artificial Intelligence, pages 193200, Berlin, Heidelberg. Springer Berlin Heidelberg.

Stein, A., Eymüller, C., Rauh, D., Tomforde, S., and Hähner, J. (2016). Interpolation-based classifier generation in XCSF. In 2016 IEEE Congress on Evolutionary Computation (CEC), pages 3990-3998.

Stein, A., Maier, R., Rosenbauer, L., and Hähner, J. (2020). XCS Classifier System with Experience Replay. In
Proceedings of the Genetic and Evolutionary Computation Conference Companion, GECCO '20.

Stein, A., Menssen, S., and Hähner, J. (2018). What about Interpolation? A Radial Basis Function Approach to Classifier Prediction Modeling in XCSF. In Proceedings of the Genetic and Evolutionary Computation Conference, GECCO '18, page 537-544, New York, NY, USA. Association for Computing Machinery.

Stein, A., Rudolph, S., Tomforde, S., and Hähner, J. (2017). Self-Learning Smart Cameras - Harnessing the Generalization Capability of XCS.

Tomforde, S., Prothmann, H., Rochner, F., Branke, J., Hähner, J., Müller-Schloer, C., and Schmeck, H. (2008). Decentralised Progressive Signal Systems for Organic Traffic Control. pages 413-422.

Urbanowicz, R. J. and Browne, W. N. (2017). Introduction to Learning Classifier Systems. Springer Publishing Company, Incorporated, 1st edition.

von Pilchau, W. P., Stein, A., and Hähner, J. (2020). Bootstrapping a DQN Replay Memory with Synthetic Experiences. ArXiv, abs/2002.01370.

Wilson, S. (2002). Classifiers that Approximate Functions. Natural Computing, 1:1-2.

Wilson, S. W. (1995). Classifier Fitness Based on Accuracy. Evolutionary Computation, 3(2):149-175.

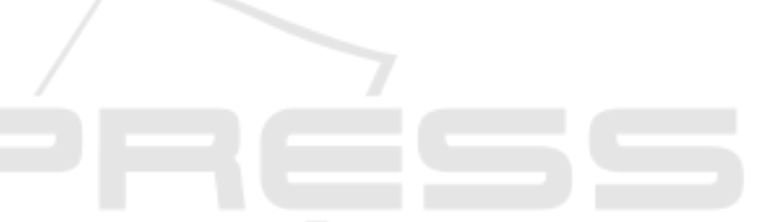

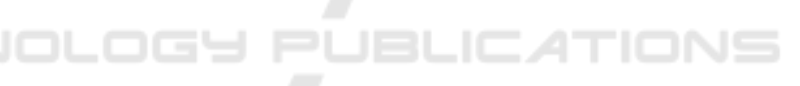

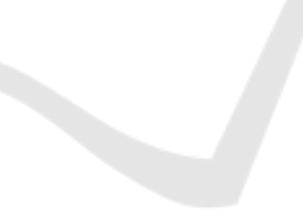

